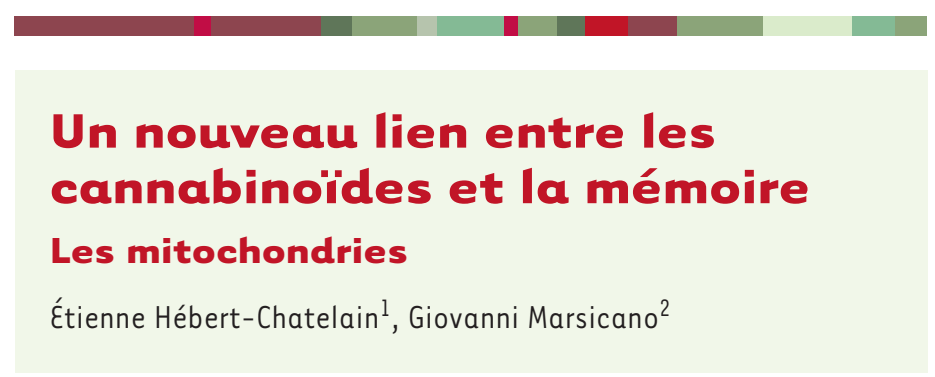

Les centrales énergétiques du cerveau Les mitochondries sont des organites qui transforment la majeure partie de l'énergie contenue dans les nutriments (glucides, lipides et protéines) en une molécule utilisable par la cellule: l'adénosine triphosphate ou ATP. Ce processus est tout particulièrement important pour le cerveau car cet organe est hautement énergivore: bien qu'il ne représente que $2 \%$ de la masse totale du corps, le cerveau humain consomme plus de $20 \%$ de l'apport énergétique total du corps humain. Le métabolisme mitochondrial s'avère notamment crucial pour assurer la transmission synaptique et la libération des neurotransmetteurs entre les neurones, le maintien des potentiels membranaires et la propagation des influx nerveux [1]. Les mitochondries sont impliquées dans plusieurs autres processus physiologiques importants. Elles produisent d'importantes quantités d'espèces réactives de l'oxygène qui jouent un rôle dans la signalisation cellulaire tout en ayant la capacité d'endommager les structures cellulaires si elles sont produites en trop grande quantité. Elles sont aussi impliquées dans l'homéostasie calcique et la mort cellulaire par apoptose. II n'est donc pas surprenant que tout défaut dans les fonctions de ces organites puisse induire une multitude de pathologies, incluant les maladies neurodégénératives et neuropsychiatriques. L'étude des processus régulant les fonctions mitochondriales nous permettra donc de mieux comprendre la physiologie du cerveau.
Les cannabinoïdes et le cerveau

Le cannabis est une plante dont les propriétés psychotropes sont connues depuis l'antiquité. Le composé psychoactif le plus connu de cette plante se nomme le $\Delta 9$-tétrahydrocannabinol du cerveau et le comportement des individus en se liant et en activant les récepteurs des cannabinoïdes ${ }^{1}$ du cerveau, principalement le récepteur $\mathrm{CB}_{1}$ (cannabinoid receptor type 1) [2] $(\rightarrow)$. Ces récepteurs sont fortement exprimés à la surface des cellules du système nerveux central et sont impliqués dans plusieurs processus physiologiques tels l'appétit, I'humeur, l'apprentissage et la mémoire [3]. L'activation du récepteur $\mathrm{CB}_{1}$ par les cannabinoïdes entraîne différentes cascades de réactions biochimiques à l'intérieur des cellules du cerveau modifiant ainsi leurs propriétés, la physiologie du cerveau et le comportement de la personne.

\section{Le $\mathrm{CB}_{1}$ mitochondrial ( $m \mathrm{tCB}_{1}$ )}

Il y a quelques années, notre équipe a découvert que le récepteur $\mathrm{CB}_{1}$ est aussi présent à la membrane des mitochondries $[4,5]$. L'activation de ce récepteur $\mathrm{CB}_{1}$ mitochondrial (nommé $\mathrm{mtC} \mathrm{B}_{1}$ ) diminue l'activité mitochondriale et la production d'ATP $[4,5]$. Étant donné l'impact direct des mitochondries sur la

\footnotetext{
${ }^{1}$ Les récepteurs des cannabinoïdes font partie des récepteurs membranaires couplés aux protéines $G$. Deux types de récepteurs sont principalement connus, $\mathrm{CB}_{1}$ et $\mathrm{CB}_{2}$ (cannabinoid receptor type 1/2), mais seul le premier serait présent dans le cerveau.
} (THC). Le THC altère le fonctionnement
${ }^{1}$ Université de Moncton, département de biologie, 18 avenue Antonine Maillet, દlA3દ9 Moncton, NB, Canada ;

${ }^{2}$ Inserm U862, Neurocentre Magendie, université de Bordeaux, 146 rue Léo Saignat, 33076 Bordeaux, France.

etienne.hebert.chatelain@umoncton.ca

physiologie du cerveau et le comportement, le $\mathrm{mtCB}_{1}$ pourrait donc constituer un régulateur important du fonctionnement de celui-ci. Nous avons ainsi initié des travaux visant à déterminer le rôle du $\mathrm{mtCB}_{1}$ parmi tous les effets des cannabinoïdes sur le cerveau.

Une analyse in silico de la séquence d'acides aminés du récepteur $\mathrm{CB}_{1}$ a permis de mieux comprendre les mécanismes de son importation dans la mitochondrie. Nous avons découvert qu'une région particulière de la protéine est nécessaire pour son adressage à la mitochondrie. Différents algorithmes ont en effet calculé que la probabilité que $\mathrm{CB}_{1}$ soit présent dans la mitochondrie est de 40 à $45 \%$ pour un récepteur «complet», alors qu'elle diminue et atteint seulement entre 1 et $3 \%$ lorsque les 22 premiers acides aminés de l'extrémité $\mathrm{N}$-terminale de la protéine sont supprimés [6]. Des virus exprimant des récepteurs $C B_{1}$ normaux et des récepteurs mutants dépourvus de ces 22 acides aminés (nommés DN22-CB ${ }_{1}$ ) ont été produits. Ces virus ont ensuite été injectés dans l'hippocampe, une région du cerveau impliquée dans la mémoire et l'apprentissage, de souris déficientes pour le gène codant le récepteur $\left(C B_{1}^{-/-}\right)$, afin de comparer la distribution cellulaire de ces deux récepteurs exogènes. Les hippocampes des souris ont ensuite été prélevés et préparés pour être observés par microscopie électronique. L'analyse de ces tissus a confirmé que ces 22 acides aminés agissent comme un signal pour adresser le récepteur $C B_{1}$ à la mitochondrie, aucune protéine $m t C B_{1}$ n'ayant été détectée chez les 


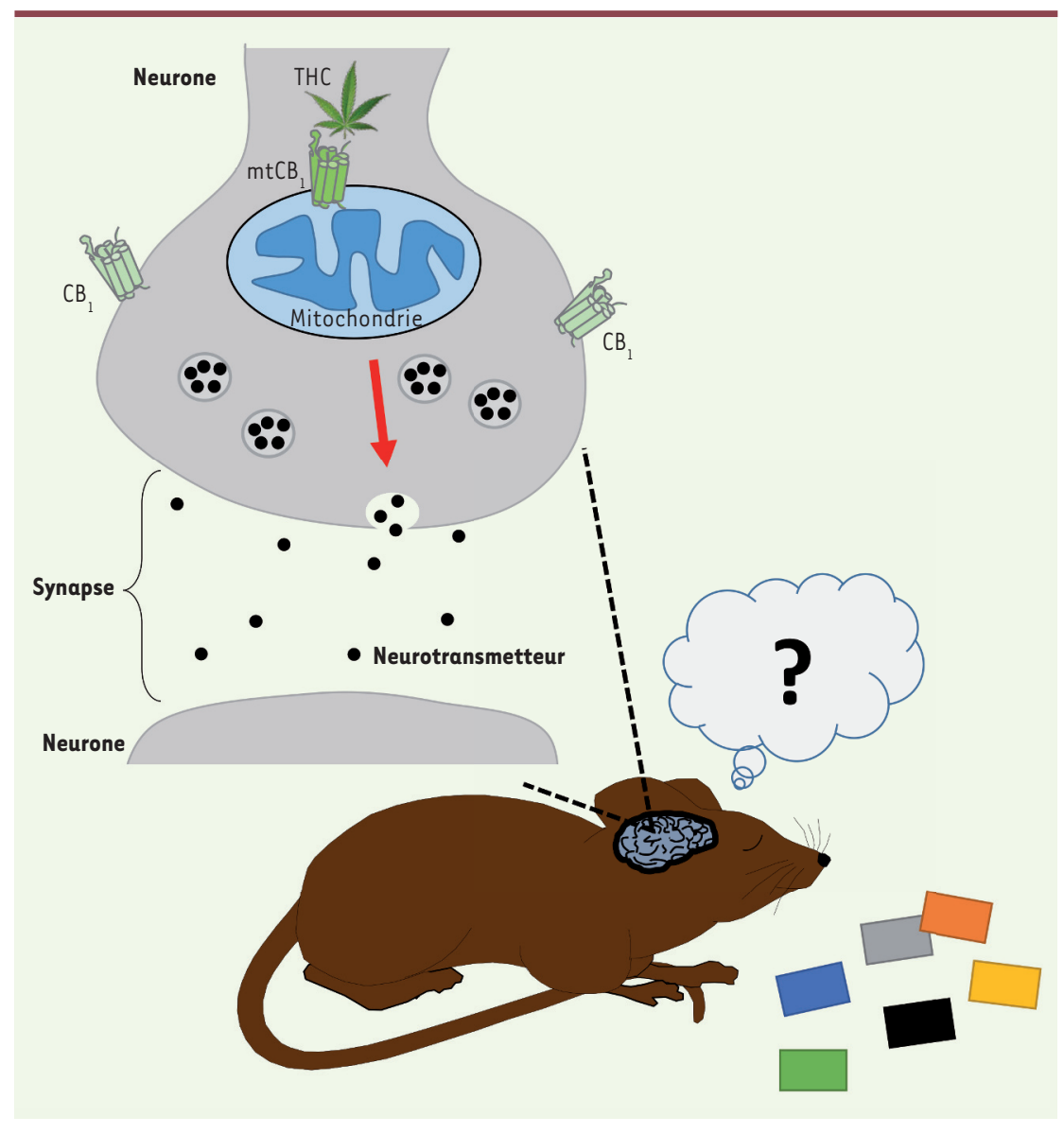

souris exprimant le récepteur tronqué DN22-CB $B_{1}$ 6]. Un premier outil capital dans notre étude du rôle spécifique des récepteurs mitochondriaux $m t C B_{1}$ avait donc été identifié.

\section{Rôle du mtCB $\mathrm{CB}_{1}$ dans la pharmacologie} des cannabinoïdes

L'expression du récepteur mutant DN22-CB 1 dans différents modèles in vitro et in vivo a par la suite permis de mieux comprendre le rôle fonctionnel de la protéine mitochondriale $\mathrm{mtCB}_{1}$ dans les effets pharmacologiques des cannabinoïdes, en particulier sur le métabolisme mitochondrial, la transmission synaptique et la mémoire.

L'analyse de modèles exprimant le récepteur mutant $D N 22-C B_{1}$ indique que le métabolisme mitochondrial n'est pas modifié dans des cellules ou des tissus traités avec des cannabinoïdes quand le récepteur $m t C B_{1}$ est absent [6]. L'acti-
Figure 1. Les cannabinoïdes affectent la mémoire en ralentissant l'activité mitochondriale. Les récepteurs des cannabinoïdes sont présents à la surface des membranes plasmiques (nommés $\mathrm{CB}_{1}$ ) et mitochondriales (nommés $\mathrm{mtCB}_{1}$ ). L'activation du $\mathrm{mtCB}_{1}$ par le THC ( $\Delta 9$-tétrahydrocannabinol, le principal composé psychoactif de la plante Cannabis sativa) entraîne une diminution du métabolisme mitochondrial. L'énergie fournie par les mitochondries est essentielle à l'activité des neurones; une diminution de l'activité mitochondriale est donc associée à une réduction de la propagation des influx nerveux et de la transmission synaptique entre les neurones de régions particulières du système nerveux central, notamment de l'hippocampe, une région impliquée dans les processus de mémoire. Ce changement dans l'activité neuronale explique pourquoi le composé THC diminue les performances mnésiques.

une baisse de la mémoire de travail ${ }^{2}$ [7]. Sachant que des déficits mitochondriaux chroniques dans le cerveau sont associés à différents troubles cognitifs, nous avons émis l'hypothèse que la diminution de l'activité mitochondriale liée au récepteur $m t \mathrm{CB}_{1}$ pouvait affecter les performances de mémoire. Un test de reconnaissance d'un nouvel objet (novel object recognition test) a été utilisé. Dans ce test, des souris sont en présence de deux objets pendant un temps donné. Durant cette phase, les souris explorent et se familiarisent avec ces objets. Après cette période d'apprentissage, on remplace l'un des deux objets par un autre objet et le comportement des souris est alors observé. En l'absence de traitement, les souris préfèrent explorer le nouvel objet et délaissent l'objet familier, démontrant qu'elles ont au préalable mémorisé cet objet familier. Lorsqu'une souris est traitée avec des cannabinoïdes après la phase d'apprentissage, on observe une baisse de mémoire.

Le rôle de $\mathrm{mtCB}_{1}$ a ensuite été exploré spécifiquement dans les processus de mémorisation. Les cannabinoïdes, en activant le récepteur $C B_{1}$, entraînent

\footnotetext{
${ }^{2}$ La mémoire de travail est la capacité de retenir et traiter l'information durant le raisonnement et l'apprentissage, et ce, de façon transitoire.
} 
de ses performances de mémoire: elle n'est plus en mesure de reconnaître l'objet auquel elle a déjà été exposée et passe pratiquement autant de temps à explorer les deux objets. Chez les souris déficientes en récepteur $C B_{1}$, les cannabinoïdes n'ont plus d'effet dans ce test mnésique ${ }^{3}$, ce qui démontre que l'altération de la mémoire par les cannabinoïdes est réellement due à l'activation des récepteurs des cannabinoïdes [6]. Afin de comprendre le rôle du récepteur mitochondrial $\mathrm{mtCB}_{1}$ dans ce processus, nous avons évalué les performances de mémoire chez des souris ne l'exprimant pas. Étonnamment, les cannabinoïdes n'affectent pas la mémoire chez les souris exprimant le récepteur mutant DN22- $\mathrm{CB}_{1}$ qui ne présentent donc pas de récepteurs mitochondriaux des cannabinoïdes dans leur hippocampe [6].

\section{Conclusion et perspectives}

On sait depuis longtemps que des dysfonctions mitochondriales chroniques sont associées à des problèmes cognitifs, mais nos résultats sont les premiers à suggérer l'importance de changements fins, ponctuels et aigus dans le fonctionnement du cerveau et le comportement. Ces résultats suggèrent que les cannabinoïdes sont en mesure d'activer le récepteur $m t C B_{1}$, de diminuer le

\footnotetext{
${ }^{3}$ Mnésique : qui a trait à la mémoire.
}

métabolisme mitochondrial, et d'altérer la physiologie et les fonctions du cerveau. C'est donc en perturbant l'activité mitochondriale que les cannabinoïdes affectent la mémoire, comme schématisé sur la Figure 1. Bien que nous n'ayons toujours pas élucidé complètement cette question, on peut émettre I'hypothèse que l'activation du récepteur $\mathrm{mtCB}_{1}$ entraînerait une baisse de la production d'ATP. $\varepsilon$ n produisant moins d'ATP, les cellules du cerveau disposeraient de moins d'énergie pour effectuer les tâches importantes, notamment la propagation des influx nerveux et les processus de plasticité synaptique qui sont impliqués dans la formation des représentations mnésiques. L'étude du récepteur mitochondrial $m t C B_{1}$ ouvre donc plusieurs nouveaux champs de recherche concernant son rôle dans les nombreux effets physiologiques des cannabinoïdes et la fonction des mitochondries dans la physiologie du cerveau.

Depuis quelques années, on découvre de plus en plus de propriétés thérapeutiques aux cannabinoïdes [7] $(\rightarrow)$. Des vertus médicales pour traiter diverses patholo-

$(\rightarrow)$ Voir la Synthèse de F. Teixeira-Clerc, $m / s n^{\circ} 8-9$, aoûtseptembre 2006, page 683 gies, du cancer à la maladie d'Alzheimer, leur sont associées. L'administration de ces molécules est cependant sou- vent accompagnée d'effets secondaires indésirables. Les travaux présentés ici laissent entrevoir la possibilité de générer des molécules aptes à activer différentes sous-populations intracellulaires de récepteurs des cannabinoïdes, et donc à proposer des traitements ciblés, sans effet secondaire. $\diamond$

Mitochondria link between

cannabinoid and memory

\section{LIENS D'INTÉRÊT}

Les auteurs déclarent n'avoir aucun lien d'intérêt concernant les données publiées dans cet article.

\section{RÉFÉRENCES}

1. Venance L, Maldonado R, Manzoni O. Le système endocannabinoïde central. Med Sci (Paris) $2004 ; 20$ : 45-53.

2. Mattson MP, Gleichmann M, Cheng A. Mitochondria in neuroplasticity and neurological disorders. Neuron $2008 ; 60: 748-66$.

3. Kano M, Ohno-Shosaku T, Hashimotodani Y, et al. Endocannabinoid-mediated control of synaptic transmission. Physiol Rev 2009 ; 89 : 309-80.

4. Benard G, Massa F, Puente N, et al. Mitochondrial $\mathrm{CB}(1)$ receptors regulate neuronal energy metabolism. Nat Neurosci 2012 ; 15 : 558-64.

5. Hebert-Chatelain $\varepsilon$, Reguero L, Puente N, et al. Cannabinoid control of brain bioenergetics: Exploring the subcellular localization of the CB1 receptor. Mol Metab $2014 ; 3$ : 495-504.

6. Hebert-Chatelain $\varepsilon$, Desprez T, Serrat R, et al. A cannabinoid link between mitochondria and memory. Nature 2016 ; 539 : 555-9.

7. Puighermanal $\varepsilon$, Marsicano G, Busquets-Garcia A, et al. Cannabinoid modulation of hippocampal longterm memory is mediated by mTOR signaling. Nat Neurosci 2009; 12 : 1152-8.

8. Teixeira-Clerc F, Julien B, Grenard P, et al. Les antagonists du récepteur $C B 1$ des cannabinoïdes : une nouvelle approche pour le traitement de la fibrose hépatique. Med Sci (Paris) 2006 ; 22 : 683-5.

\section{LA FONDATION PREMUP : UN OPÉRATEUR DE TERRAIN EN PÉRINATALITÉ RECONNU POUR SON EXCELLENCE ET SON INTERDISCIPLINARITÉ}

La Fondation de coopération scientifique PremUp, unique en Europe, intervient sur la prévention du handicap à la naissance, par la protection de la santé de la femme enceinte et du nouveau-né.

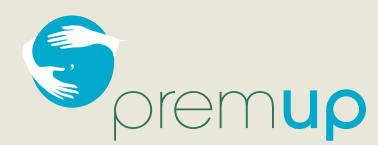

FONDATION DE COOPÉRATION SCIENTIFIQUE SUR LA GROSSESSE ET LA PRÉMATURITÉ

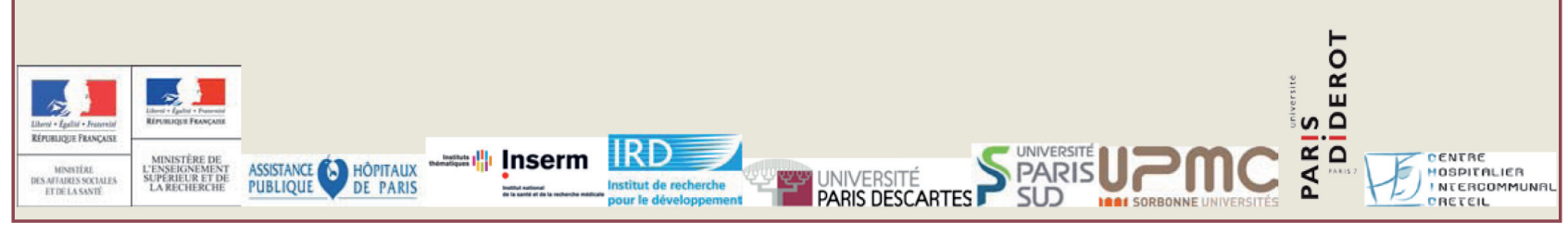

